\title{
CREATIVITY INC.: OVERCOMING THE UNSEEN FORCES THAT STAND IN THE WAY OF TRUE INSPIRATION
}

\author{
Authors: Ed Catmull and Amy Wallace
}

I have a confession to make..... I am a huge fan of Pixar Movies... Who isn'tffffffl That is how I came to read this awesome book. I wanted to learn about the foundation of Pixar and its work culture, which is quite nicely detailed in the book.

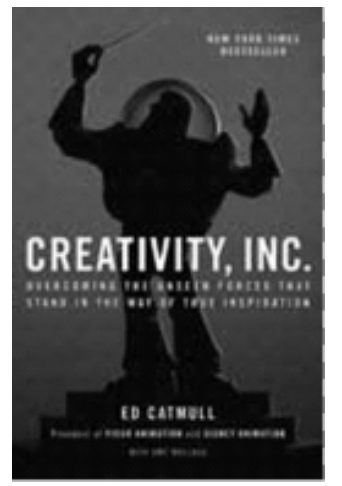

Ed Catmull, for those who don't recognise the name is one of the founders of Pixar and it's current President. Amy Wallace is an editor at Los Angeles magazine as well as a correspondent for GQ.

After toiling away in the emerging field of computer sciences for some twenty years, Ed eventually succeeded in accomplishing his life's goal: to make the first, full-length animated feature film. After successful completion of his life's goal, he came up with a new goal: to create a viable, sustainable creative business that could last without the input of himself or the other creative powers that be at Pixar. This book essentially encapsulates the lessons he's learned that have not only made Pixar "work" but will hopefully continue to do so for years to come.
He shares the story of his early days in computer graphics and his uncertain transition into management, giving specific examples of things that worked and did not work. There are numerous anecdotes about his interactions with Steve Jobs and the various directors at Pixar, and deep reflection on the roles of personality, pride, bias, objectivity, failure, success, teamwork and the various permutations thereof.

For nearly twenty years, Pixar has dominated the world of animation, producing such beloved films as the Toy Story trilogy, Monsters, Inc., Finding Nemo, The Incredibles, Up, and WALL-E, which have gone on to set box-office records and garner thirty Academy Awards. The joyousness of the storytelling, the inventive plots, the emotional authenticity: In some ways, Pixar movies are an object lesson in what creativity really is.

Here, in this book, Catmull reveals the ideals and techniques that have made Pixar so widely admired-and so profitable. The essential ingredient in Pixar movies' success is the unique environment that Catmull and his colleagues built at Pixar - Encouraging and nurturing Innovation.

Success often leads to complacency, what-

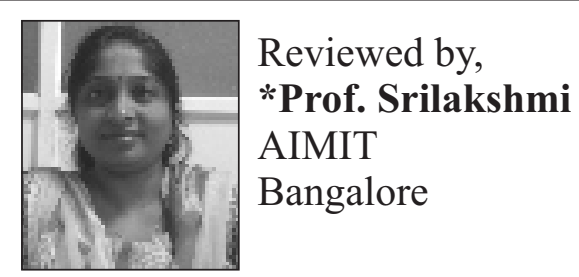


worked-before is encoded as rule and takes away the flexibility to innovate, population and budgets get blown out of proportion until the constraints that provided inspiration disappear, and as a result creative streaks tend to be frustratingly unsustainable. This book teaches us how we can avoid this trap and be creative all through. The way they have built a staff culture that pushes against the status quo allowing them to pursue new and innovative ideas is inspiring. This book helps unlock the secret to how they achieve this and how they empower leaders to accomplish it.

I felt the book is divided into two parts. Roughly a third of this book is related to stories about Pixar the company and their movie products, while the remaining two thirds are stories about how creativity is nurtured there. The creativity stories are of interest, and Catmull earnestly shares details of company meetings, programs, and policies that were tried, as well as his personal thoughts about how things should work.

One of the great traditions at Pixar that Catmull is most proud of is the concept of the "Braintrust". Early in the film-making process, they hold a screening with employees who are tasked with giving candid feedback to the Director. These employees come from all parts of Pixar and are collectively referred to as the Braintrust. The great part about the Braintrust is that the Director is required to listen to the feedback, but is not required to incorporate it all into the movie. This prevents the Braintrust from becoming a power-house that can eventually be corrupted. The nice thing about Pixar is that the Braintrust has employees from different levels in the organization and they have created an atmosphere where there is no fear of retribution if the feedback is not favorably received by the Director.

Similarly another unique experiment at Pixar is the 'Notes-day' in 2013 when the company halted all operations and the entire staff spent the day working with each other in teams and giving their feedback about the company.Notes Day was invaluable for the company, as staff members felt free to engage in an open dialogue about the issues they faced, meaning that problems were shared and solved.

Several ancedotes really touched my heart. Just to name one was the Toy Story-2 issue. The Pixar founders" "passion for excellence," for example, had a major positive impact on their work. The company's employees always strive to do their very best, and thus and go the extra mile to achieve their lofty goal.Several serious problems arose during the production of 'Toy Story 2' that threatened to ruin their chances of success. But because everyone at Pixar shared the goal of achieving excellence, they worked around the clock seven days a week to resolve these problems. The result: a fantastic film that grossed over $\$ 500$ million at the box office.

Another culture at Pixar that is very impressive is they accept that mistakes are part of the process and try to weed them out with each new iteration of their projects. Central to this philosophy is the idea that the whole team, rather than just a single individual, is responsible for failure, so everyone works together to overcome it. Catmull cites the many problems that arose during the production of Monsters Inc., the first film Pixar made without its most experienced director. In spite of this, the team kept at it, tackling the problems over and over again until they got it right. 
Furthermore to nurture creativity, workers have control over their workspace - anyone can decorate their workspace however they want, no matter how elaborate, as an expression of their unique personality. Alongwith it,two days every month is "personal project days," where employees can use all the available technology to work on any project or problem that they find personally interesting. By giving them time and resources, Pixar ensures that their employees will both remain happy and possibly even come up with some creative ideas that could benefit the company! This Catmull says was the fruit of experience at Utah University in 1960s itself where the students are allowed to pursue any area of interest.
My main management take-aways are that you need to trust your employees, encourage candor, and make them feel safe enough to take risks and fail. In conclusion, Creativity, Inc. explores the peaks and troughs in the history of Pixar along with Ed Catmull's personal journey towards becoming the successful manager he is today. In doing so, he has explained the management beliefs he has acquired along the way, and has offered actionable advice on how to turn any team members into creative superstars. 